\title{
Mitteilungen der DVPW-Sektion »Internationale Politik«
}

\author{
1. Workshop der Sektion: »IR Meets Didactics: Die neue Lehre der \\ Internationalen Beziehungen ", Akademie für Politische Bildung in Tutzing, \\ 19./20. März 2015
}

Die Sektion veranstaltete am 19./20. März 2015 in Tutzing einen Workshop zu Fragen rund um die Lehre in den Internationalen Beziehungen (Das Tagungsprogramm ist auf der Website der IP-Sektion einzusehen). Die Tagung fand mit finanzieller Unterstützung der Akademie für Politische Bildung in Tutzing statt. Der Workshop sollte den Erfahrungsaustausch zwischen Hochschuldidaktiker/-innen und Politikwissenschaftler/-innen aus dem Bereich der Internationalen Beziehungen (IB) befördern und best practices der Lehre in den IB erarbeiten. Der Workshop reagierte damit auf den oft beklagten, aber selten bekämpften Mangel des auf die Lehre bezogenen Austausches zwischen akademischen Lehrkräften. Während der Austausch über Forschungsfragen in den IB (wie auch in anderen akademischen Disziplinen) bestens institutionalisiert ist, bleibt ein vergleichbarer Austausch über Lehrfragen völlig unterentwickelt. Deshalb »zimmern« sich akademische Lehrkräfte ihre eigene Lehre zumeist selbst zurecht. Der Workshop sollte hier Abhilfe schaffen, indem unter Beteiligung von Hochschuldidaktiker/-innen ein Erfahrungsaustausch zwischen engagierten akademischen Lehrern organisiert wird. Die Tagung hatte knapp 30 Teilnehmer/-innen. Zum Redaktionsschluss der ZIB hat die Tagung noch nicht stattgefunden. Die Sektion wird einen kurzen Konferenzbericht auf ihrer Website veröffentlichen.

\section{Veranstaltungen der Sektion auf dem DVPW-Kongress, 21.-25. September 2015}

Die Sektion wird auf dem DVPW-Kongress an der Universität Duisburg-Essen am Dienstag und am Mittwochnachmittag insgesamt drei Panels anbieten. Die Panels, die unterschiedliche Aspekte des Kongressthemas »Vorsicht Sicherheit! Legitimationsprobleme der Ordnung von Freiheit« aufgreifen sollten, wurden durch einen Call for Panels im Vorfeld eingeworben. Besonders erfreulich ist die große Resonanz auf diesen Call, die aufgrund der unglücklichen Terminkollision zwischen dem DVPW-Kongress und der 9. Pan-European Conference on International Relations in Sizilien (siehe Punkt 5) nicht selbstverständlich war.

Neben den drei Panels wird die IP-Sektion am Donnerstagnachmittag einen Roundtable zum Thema »Geheimnis in der Außenpolitik: zwischen Staatsräson und öffentlicher Kontrolle« gemeinsam mit der Sektion für Politische Theorie und Ideengeschichte veranstalten. Einzelheiten zum Programm der IP-Sektion sind der Web- 
site der DVPW (www.dvpw.de) sowie der Website der IP-Sektion (www.unibw.de/ib-sektion) zu entnehmen.

\section{Mitgliederversammlung der Sektion während des DVPW-Kongresses}

Ebenfalls im Rahmen des DVPW-Kongresses findet am Mittwoch, den 23. September 2015 von 16.30 Uhr bis 17.30 Uhr die Mitgliederversammlung der Sektion statt (Ort siehe Kongressprogramm). Dabei steht neben den Berichten über die Aktivitäten der Sektion die Wahl der Sektionssprecher/-innen an. Vorschläge dazu wie auch zu weiteren Tagesordnungspunkten können bis zum 1. August 2015 an Anna Geis (anna.geis@ovgu.de) gerichtet werden. Das Sektionssprecherteam bittet die Mitglieder der Sektion auch um rege Beteiligung an der DVPW-Mitgliederversammlung, in der u. a. Vorstand und Beirat der DVPW neu gewählt werden.

\section{Auswahl des »Besten Papiers« im Rahmen der 4. Offenen Sektionstagung in Magdeburg, 25.-27. September 2014}

Auf der 4. Offenen Sektionstagung der IP-Sektion wurde erstmalig ein Preis für das »Beste Papier der Sektionstagung« ausgelobt, das in der Zeitschrift für Internationale Beziehungen (ZIB) veröffentlicht werden soll. Die Leiter/-innen und Diskutanten/-innen der Panels wurden gebeten, hierzu Vorschläge beim Sektionssprecherteam einzureichen. Aus den eingereichten Vorschlägen hat das Sektionssprecherteam drei Papiere ausgewählt, die dann durch drei anonyme Gutachter/-innen begutachtet wurden. Die Begutachtung entsprach den üblichen strengen Standards der ZIB. Auf Grundlage eines einhelligen Urteils der drei Gutachter/-innen, dem sich das Sektionssprecherteam anschloss, wurde das Papier von Dr. Simon Koschut (Universität Nürnberg/Erlangen) mit dem Titel »Macht der Gefühle: Zur Bedeutung von Emotionen für die sozialkonstruktivistische Diskursforschung in den IB« zum »Besten Papier« der 4. Offenen Sektionstagung 2014 in Magdeburg bestimmt. Der Beitrag wird im Heft 2/2015 der ZIB erscheinen.

\section{EISA-Tagung in Giardini-Naxos/Sizilien, 23.-26. September 2015}

Zum nunmehr neunten Mal wird 2015 die Pan-European Conference on International Relations vom 23. bis 26. September 2015 in Giardini-Naxos (Sizilien) stattfinden, organisiert von der European International Studies Association (EISA) (bis zur Konferenz in Warschau 2013 war die ECPR Standing Group on International Relations für diese Konferenzen verantwortlich). Unter dem Dachthema »The Worlds of Violence« wird die Konferenz über 60 Sektionen versammeln und damit eine der größten europäischen Konferenzen überhaupt werden. Erfreulicherweise werden auch zahlreiche deutsche Fachkollegen/-innen daran teilnehmen. Wie be- 
reits oben erwähnt, ist die Terminkollision mit dem DVPW-Kongress allerdings sehr unglücklich. Mögliche Terminüberschneidungen zwischen großen Kongressen im europäischen Raum sollten in Zukunft intensiver im Vorfeld erkundet werden.

\section{Kontaktdaten Sektionssprecher/-innen}

Prof. Dr. Anna Geis (geschäftsführend 2014/15)

Otto-von-Guericke-Universität Magdeburg

Institut für Politikwissenschaft

Zschokkestr. 32, 39104 Magdeburg

Tel.: 0391 67166-57,E-Mail: anna.geis@ovgu.de

Prof. Dr. Stephan Stetter (geschäftsführend 2012/13)

Institut für Politikwissenschaft

Universität der Bundeswehr München

Werner-Heisenberg-Weg 39, 85577 Neubiberg

Tel.: 089 6004-3175, E-Mail: stephan.stetter@unibw.de

Prof. Dr. Bernhard Zangl (geschäftsführend 2013/14)

Ludwig-Maximilians-Universität München

Geschwister-Scholl-Institut für Politikwissenschaft

Lehrstuhl für Global Governance

Oettingenstr. 67, 80538 München

Tel.: 089 2180-9018, E-Mail: Bernhard.Zang1@gsi.uni-muenchen.de

\section{Kontaktdaten Nachwuchssprecher/-innen}

Dr. Andreas Kruck

Ludwig-Maximilians-Universität München

Geschwister-Scholl-Institut für Politikwissenschaft

Oettingenstraße 67, 80538 München

Tel.: 0892180 9069, E-Mail: andreas.kruck@gsi.uni-muenchen.de

Dr. Gabi Schlag

Otto-von-Guericke-Universität Magdeburg

Institut für Politikwissenschaft

Zschokkestr. 32, 39104 Magdeburg

Tel.: 0391 67568-04, E-Mail: gabi.schlag@ovgu.de 